\title{
Melphalan Hydrochloride/Sulfobutyl Ether Beta-Cyclodextrin Complex
}

National Cancer Institute

\section{Source}

National Cancer Institute. Melphalan Hydrochloride/Sulfobutyl Ether Beta-Cyclodextrin

Complex. NCI Thesaurus. Code C84878.

A propylene glycol-free intravenous formulation containing the hydrochloride salt of the nitrogen mustard phenylalanine derivative melphalan complexed with polyanionic sulfobutyl ether beta-cyclodextrin (SBE-CD) with potential antineoplastic activity. Upon administration, melphalan is converted into highly reactive ethylenimmonium intermediates that induce covalent guanine N7-N7 intra- and inter-crosslinks and alkylation of adenine N3 of DNA; RNA and proteins may also be alkylated. Subsequently, RNA transcription and protein synthesis are inhibited, resulting in cell growth arrest. The addition of sulfobutyl ether beta-cyclodextrin to the formulation improves the solubility, stability and ease of use of melphalan; cyclodextrins are cyclic dextrins derived from starch. 